Article

\title{
Optical Spectra Tuning of All-Glass Photonic Bandgap Fiber Infiltrated with Silver Fast-Ion-Conducting Glasses
}

\section{Ioannis Konidakis * and Stavros Pissadakis}

Foundation for Research and Technology-Hellas (FORTH), Institute of Electronic Structure and Laser (IESL), P.O. Box 1385, 71110 Heraklion, Greece; E-Mail: pissas@iesl.forth.gr

* Author to whom correspondence should be addressed; E-Mail: ikonid@iesl.forth.gr; Tel.: +30-281-039-1835; Fax: +30-281-039-1318.

Received: 2 July 2014; in revised form: 3 August 2014 / Accepted: 4 August 2014 /

Published: 7 August 2014

\begin{abstract}
Silver iodide metaphosphate glasses of the $x \mathrm{AgI}+(1-x) \mathrm{AgPO}_{3}$ family are embedded inside the air capillaries of a commercial silica photonic crystal fiber (PCF) by means of vacuum-assisted infiltration technique. In this paper, we report on tuning the photonic bandgap (PBG) guidance characteristics of the fabricated all-glass photonic bandgap fibers, by varying the composition of the fast-ion-conducting phosphate glass infiltration medium. Doping $\mathrm{AgPO}_{3}$ metaphosphate glass with $\mathrm{AgI}$ significantly alters the PBG guidance patterns in the examined range between 350 and $1750 \mathrm{~nm}$, as it leads to the introduction of numerous additional transmission stop-bands, while affecting scattering dependant losses. The effect of phosphate glass cooling method during sample fabrication on the transmission behavior of the $x \mathrm{AgI}+(1-x) \mathrm{AgPO}_{3} / \mathrm{PCFs}$ is also considered.
\end{abstract}

Keywords: photonic crystal fiber; microstructured fiber design; silver phosphate glass; fast ion conducting; clusters; photonic bandgap guidance

\section{Introduction}

Photonic bandgap (PBG) optical fiber guidance relies on the existence of a refractive index contrast opto-geometrical configuration, where a low refractive index photonic crystal fiber (PCF) core is surrounded by high refractive index strands ordered in a periodic lattice, while rendering the low refractive index fiber core a phase defect of this periodic lattice wherein propagation at discrete frequencies is allowed [1-3]. This specific guidance mechanism has been also successfully described 
by antiresonant reflecting optical waveguide (ARROW) guidance theory, assuming guidance into the PCF core for light in the form of leaky modes into the high refractive index strands. Thus, by tuning the refractive index, absorption loss, and structural/scattering properties of the infiltrated media inside the PCF capillaries, the spectral signature of the PBG-PCF can be easily tuned. On this basis, the infiltration of PCFs' air capillaries with high refractive index media, such as inorganic glasses, appears promising towards the design of in-fiber light emitting and sensing devices exploiting PBG or modified total internal reflection guidance [4-8]. Recently, the fabrication of an all-glass PBG fiber by vacuum-assisted infiltration of molten silver metaphosphate $\left(\mathrm{AgPO}_{3}\right)$ glass into an air/silica PCF was demonstrated [6,7]. Initially, the effect of $\mathrm{AgPO}_{3}$ glass laser photosensitivity on the guiding properties of the composite PCF was investigated [6], whereas, the latter study focuses on shaping the PBG guiding properties of $\mathrm{AgPO}_{3}$ glass infiltrated PCFs upon introduction of silver nanoparticles inside the phosphate glass strands via thermal poling processes [7]. Along these lines, the potential exploitation of silver nanoparticles plasmon resonance characteristics attracts substantial interest in terms of the development of in-fiber electrically driven devices and sensors [9-11].

In the work presented here, we exploit the advantages of another set of functional glasses as PCF infiltrated medium, as we report on the fabrication of all-glass PBG fiber by infiltration of silver fast-ion-conducting (FIC) glasses of the system $x \mathrm{AgI}+(1-x) \mathrm{AgPO}_{3}$ into the PCF capillaries. The choice of infiltrating PCFs with FIC phosphate glasses is prompted by variety of reasons and opens up new prospective for developing new devices but also for studying interesting light propagation effects. Apart from their soft nature (extremely low glass transition temperature, $T_{\mathrm{g}}$ ) and low melt viscosities that allow the formation of highly homogeneous glass strands inside the PCFs, silver phosphate FIC glasses provide two additional advantages of great importance. Firstly, they are characterized by ionic conductivities up to $10^{-2} \mathrm{~S} \cdot \mathrm{cm}^{-1}$ at room temperature, i.e., such superior conductivity values are analogous to that of molten salt electrolytes like $\mathrm{KNO}_{3}$ used for electric battery applications, and demonstrate the high charge carrier mobility within the glass matrix [12-15]. Indicatively, the superior mobility of silver cations can be further exploited for tuning the guiding properties of the composite all-glass FIC/PCF, towards the development of in-fiber electrically driven devices [11]. Secondly, the addition of AgI leads to the formation of conduction pathways and AgI-rich microdomains or clusters which percolate though the existing phosphate glass network [14,16,17]. Such microdomains within the glass strands of the infiltrated PCFs act as centers of scattering [6,7,16], and thus, the concentration of AgI is a powerful tool for tuning the PBG guidance characteristics of the FIC glass infiltrated PCFs [18]. The easy tuning of the spectral position of the transmission permitted bands for such an all-solid PCF can be used for overlapping them with the emission bands of active ions (such as Er, $\mathrm{Yb}$ or $\mathrm{Nd}$ ), and developing lasing or amplification components. In addition, the ability to tune the scattering morphology of high transparency FIC glasses, infiltrated inside PCFs, can be used for triggering other light localization effects, in addition to the original PBG guidance. This can be of great importance since the infiltration of glasses of controlled scattering behavior into the capillaries of PCFs, constituting a photonic bandgap system with spatially confined and controlled statistics disorder, can be used for the realization of random lasing [19] and slow light devices [20]. Based on the above, we hereby report on both the effects of AgI concentration and FIC glass cooling method employed for the glass formation on the transmission patterns of composite $x \mathrm{AgI}+(1-x) \mathrm{AgPO}_{3}-\mathrm{glass} / \mathrm{PCF}$ 
samples, while all samples are also examined by scanning electron microscopy (SEM) in order to verify glass filling quality.

\section{Experimental}

\subsection{Fabrication of $x \mathrm{AgI}+(1-x) \mathrm{AgPO}_{3} / \mathrm{PCF}$ Samples}

For the experiments of the present study a commercially available all-silica PCF was used, i.e., LMA-10 drawn by NKT Photonics Ltd. (Birkerod, Denmark). The microstructured LMA-10 fiber has a periodic four-ring lattice of hollow capillaries running through the entire length of the PCF. The average diameter of the capillaries is $2.85 \mu \mathrm{m}$ as determined by scanning electron microscopy (SEM), whereas the lattice constant is found to be $6.4 \mu \mathrm{m}$. Based on the silver metaphosphate $\mathrm{AgPO}_{3}$ glass, fast-ion-conducting (FIC) glasses of the system $x \mathrm{AgI}+(1-x) \mathrm{AgPO}_{3}$ with $x=0.3$ and 0.55 were prepared by melting appropriate amounts of $\mathrm{AgI}, \mathrm{AgNO}_{3}$ and $\mathrm{NH}_{4} \mathrm{H}_{2} \mathrm{PO}_{4}$ dry powders within an electrical furnace [21,22]. All reactions were carefully monitored until gas evolution ceased. The air-capillaries of the LMA-10 fiber were filled by means of a vacuum-assisted infiltration process which is described in detail elsewhere [6]. For the fibers fabricated here, a temperature of $670{ }^{\circ} \mathrm{C}$ was maintained throughout the infiltration process. At this temperature, we achieved filling ratios of $\sim 3.5 \mathrm{~cm} \cdot \mathrm{h}^{-1}$ for the $\mathrm{AgPO}_{3}$ glass and $\sim 9 \mathrm{~cm} \cdot \mathrm{h}^{-1}$ for the two FIC $x \mathrm{AgI}+(1-x) \mathrm{AgPO}_{3}$ glasses, i.e., depending on the melt viscosity. Only two PCF samples were infiltrated from each FIC glass melt as HI gas evolution over subsequent reheating may eventually alter the nominal stoichiometry and local topology of the glasses [22]. A splat sample of the $x \mathrm{AgI}+(1-x) \mathrm{AgPO}_{3}$ glass with $x=0.55$ was sandwiched between two silica plates forming an even layer of $\sim 0.06 \mathrm{~mm}$ thickness, kept at a temperature of $\sim 150{ }^{\circ} \mathrm{C}$. Spectrophotometric measurements using a white light profilometer (FR-Basic VIS/NIR, ThetaMetrisis S.A., Athens, Greece) of this sample revealed that its short wavelength absorption band sharply starts at $\sim 450 \mathrm{~nm}$.

After the infiltration stage, two distinct cooling methods of the glass melt were followed, namely fast-cooling (f.c.) and slow-cooling (s.c.). Following the fast-cooling method, the infiltrated fiber was instantly removed from the electrical furnace and exposed to ambient room temperature. In contrast, by following the slow-cooling protocol, after the infiltration period the fiber was immerged from the glass melt with the aid of a micrometric stage; however, it remained within the electrical furnace to sustain a monitored cooling rate of $\sim 5^{\circ} \mathrm{C} \cdot \mathrm{min}^{-1}$. Scanning electron microscopy (SEM) was employed to examine the infiltration quality of the composite LMA-10 samples studied herein. SEM scans were performed in different cross-sections of the entire infiltrated length of the PFC samples in order to ensure axial homogeneity of the phosphate glass strands.

\subsection{Optical Spectra Measurements}

The transmission spectra of homogenous sections of $\mathrm{AgPO}_{3}$ and $x \mathrm{AgI}+(1-x) \mathrm{AgPO}_{3} / \mathrm{LMA}-10$ samples of $1 \mathrm{~cm}$ length were measured by using a microscope objective coupling-in/out set-up and a broadband supercontinuum source (SCS, 350-2000 nm), as described in [6]. Namely, a 25× microscope objective was used to couple light into the solid silica core, whereas a $60 \times$ objective collected the near field light from the fiber end face. An iris diaphragm was employed for solely 
selecting the fiber core guided output light, and reducing the stop-band noise lever originating from the light propagation through the phosphate glass strands of the cladding. Finally, the detected light was coupled through a multimode fiber to an optical spectrum analyzer.

\section{Results and Discussion}

\subsection{Scanning Electron Microscopy (SEM)}

Figure 1a,b show SEM scans of a cleaved cross section of $0.3 \mathrm{AgI}+0.7 \mathrm{AgPO}_{3} / \mathrm{LMA}-10$ fiber prepared following fast-cooling method. The fast-cooled $0.3 \mathrm{AgI}+0.7 \mathrm{AgPO}_{3}$ FIC glass strands appear to exhibit minimal cluster formation of average size less than $15 \mathrm{~nm}$, as determined by thorough image analysis of the SEM photos by the aid of computing software [23]. Such cluster size is comparable to the size of silver nanoparticles previously reported for the binary $\mathrm{AgPO}_{3}$ glass, i.e., prepared under equivalent conditions [7], while resting on the Rayleigh scattering regime. Inspection of Figure 1c,d reveals an average size of $\sim 350 \mathrm{~nm}$ for the clusters embedded within the slow-cooled $0.3 \mathrm{AgI}+0.7 \mathrm{AgPO}_{3}$ glass strands; $\mathrm{AgI}$-nanoparticles of this size rest on the Mie scattering regime, exhibiting angular dependent scattering efficiency.

Figure 1. (a) SEM scan of a cleaved end face of $0.3 \mathrm{AgI}+0.7 \mathrm{AgPO}_{3} / \mathrm{LMA}-10$ fiber prepared following fast-cooling method; (b) Magnified area of panel (a); (c) SEM scan of the same type of composite fiber prepared following slow-cooling method; (d) Magnified area of panel (c).
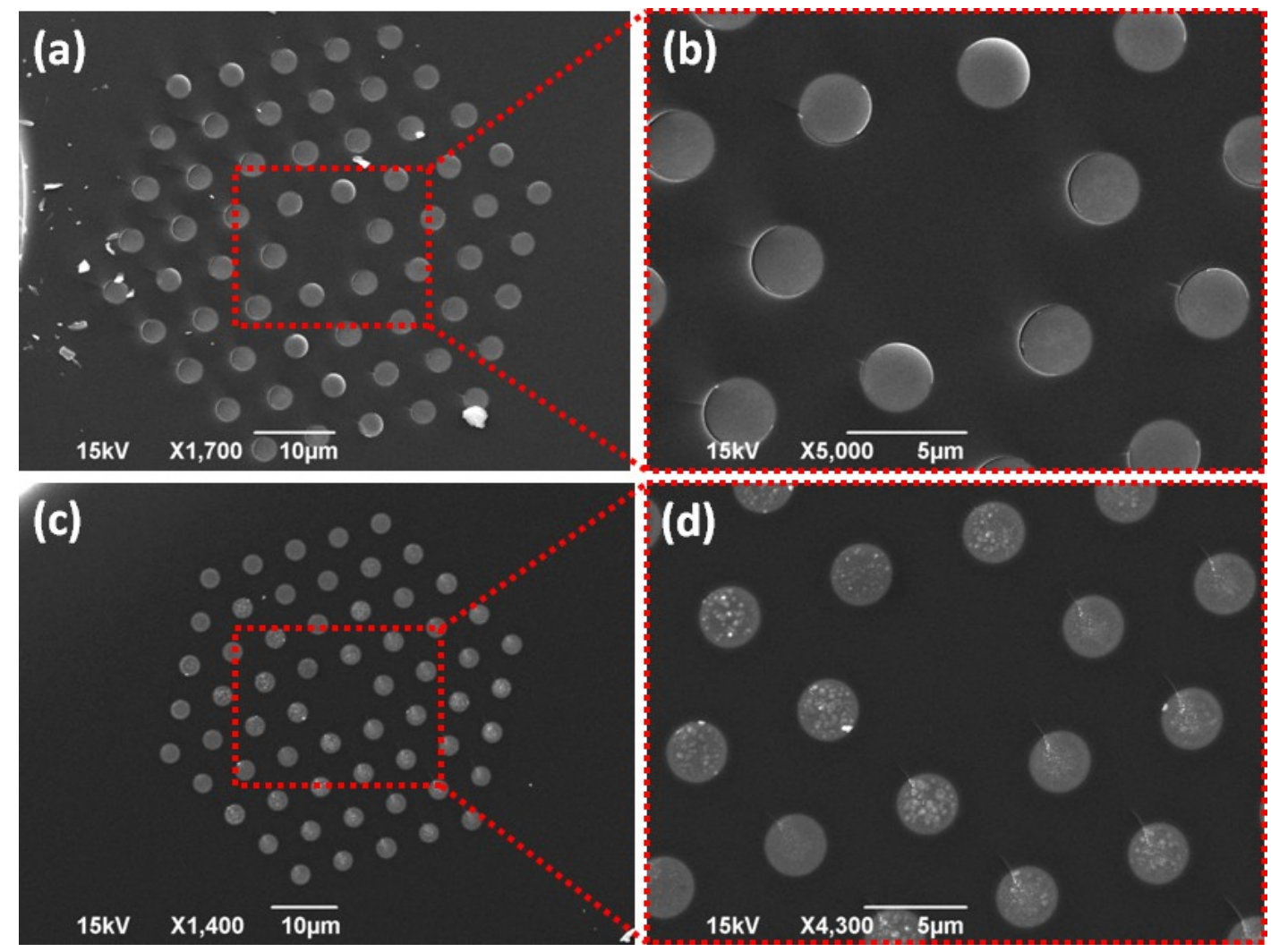

Similar SEM examination was performed on $0.55 \mathrm{AgI}+0.45 \mathrm{AgPO}_{3} / \mathrm{LMA}-10$ samples. Figure $2 \mathrm{a}, \mathrm{b}$ display details of cross section of samples fabricated following fast-cooling protocol, while 
corresponding scans for fibers of the slow-cooling protocol are presented in Figure 2c,d. Again, for the fast-cooled FIC glass clusters of negligible size of less than $15 \mathrm{~nm}$ are revealed, whereas their size increases to a figure of $\sim 150 \mathrm{~nm}$ when the slow-cooling method is followed. Overall, SEM analysis of all fabricated PCFs revealed good quality of FIC phosphate glass strands without hollow inclusions, i.e., bubbles or cracks. We note that SEM scans of the binary metaphosphate glass $\mathrm{AgPO}_{3} / \mathrm{LMA}-10$ fibers have been extensively presented in previous studies [6,7], and thus, not included herein for the sake of space.

Figure 2. (a) SEM scan of a cleaved end face of $0.55 \mathrm{AgI}+0.45 \mathrm{AgPO}_{3} / \mathrm{LMA}-10$ fiber prepared following fast-cooling method; (b) Magnified area of panel (a); (c) SEM scan of the same type of composite fiber prepared following slow-cooling method; (d) Magnified area of panel (c).
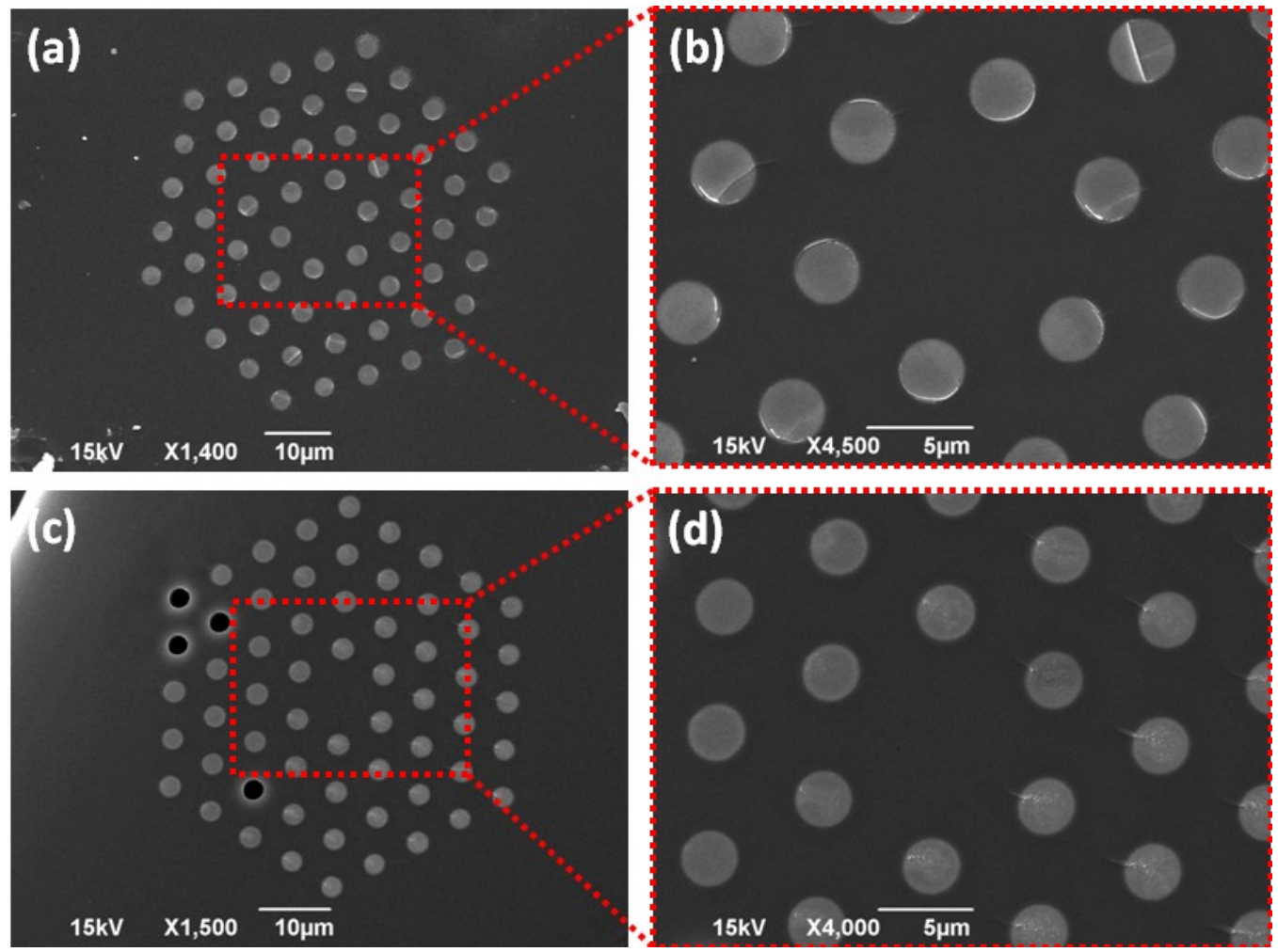

\subsection{Transmission Spectra of $\mathrm{AgPO}_{3}$ and $x \mathrm{AgI}+(1-x) \mathrm{AgPO}_{3} / \mathrm{LMA}-10$ Samples}

Figure 3 shows typical experimental transmission spectra of $1 \mathrm{~cm}$ long $x \mathrm{AgI}+(1-x) \mathrm{AgPO}_{3} / \mathrm{LMA}-10$ fibers, for $x=0,0.3$ and 0.55 , prepared following fast-cooling method. All transmission patterns demonstrate photonic bandgap (PBG) guidance with extinction ratios of $\sim 20 \mathrm{~dB} / \mathrm{cm}$ for the AgI-doped glasses, and $\sim 25 \mathrm{~dB} \cdot \mathrm{cm}^{-1}$ for the binary $\mathrm{AgPO}_{3}$ glass. Due to the higher refractive index of the AgI-doped glasses compared to the undoped, basic silver metaphosphate matrix, further optical bands are inserted into their transmission spectra; as predicted from ARROW theory greater accumulation from those bands appears at the short wavelengths regimes. The reported refractive indexes for the AgI doped glasses with respect to the AgI concentration are 1.79, 1.90 and 2.1, for $x=0,0.3$ and 0.55 respectively [24]. Moreover, the transmission losses for the AgI doped glass infiltrated PCFs appear slightly higher than those of the sample for $x=0$. This is attributed to two main reasons: (a) scattering 
induced by the favored formation of silver particles for those glass compositions on the interface between the silica PCF and the soft glass inclusion, increases overall attenuation; and (b) the greater the AgI content the higher the absorption losses of the infiltrated glass, shifting bandgap edge to longer wavelengths [25].

Figure 3. Transmission spectra of $1 \mathrm{~cm}$ long $x \mathrm{AgI}+(1-x) \mathrm{AgPO}_{3} / \mathrm{LMA}-10$ fibers, for $x=0,0.3$ and 0.55 , prepared following fast-cooling method. Insets on the right present focus on the short wavelength guiding regime between 450 and $850 \mathrm{~nm}$. All spectra are normalized to the corresponding spectrum of the pristine photonic crystal fiber (PCF).

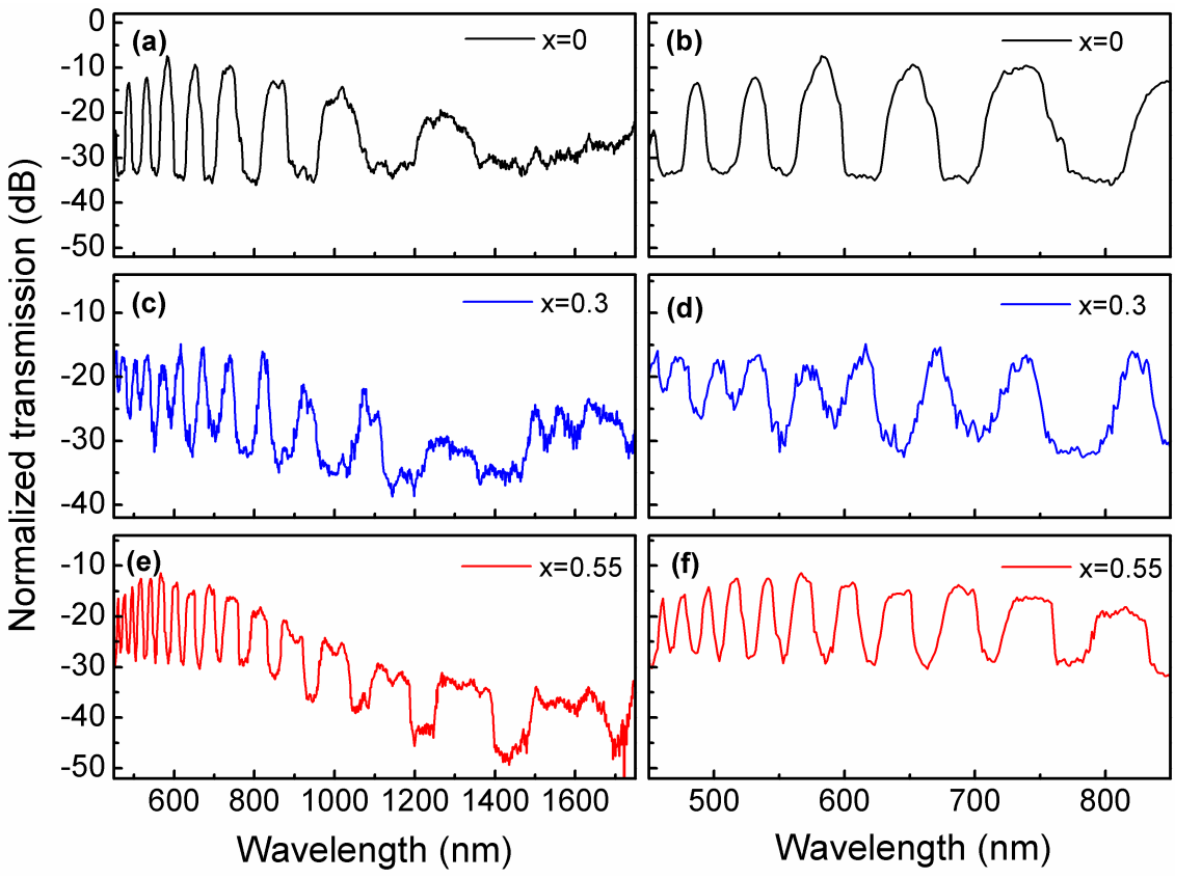

Then, the effect of the cooling method followed during sample fabrication, i.e., fast-cooling and stop-cooling, on the PBG guidance characteristics of the composite FIC-glass/LMA-10 fibers was investigated. Figure 4 presents transmission patterns for $0.3 \mathrm{AgI}+0.7 \mathrm{AgPO}_{3} / \mathrm{LMA}-10$ and $0.55 \mathrm{AgI}+0.45 \mathrm{AgPO}_{3} / \mathrm{LMA}-10$ fibers. For the first fiber (Figure $4 \mathrm{a}$ ), where $x=0.3$, by adopting the slow-cooling protocol, the overall transmission loss increases by as much as $\sim 10 \mathrm{~dB} \cdot \mathrm{cm}^{-1}$ at specific bands of transmission. Slow cooled PBG-PCF exhibits spectrally shifted transmission stopbands, with respect to the fast-cooled counterpart, indicating that even though glass strands composition of the two infiltrated PCFs is identical, refractive index dispersivity substantially differs between them. There are two significant spectral features characterizing the data of Figure $4 \mathrm{a}$ : the first refers to the extinction ratio of the transmission stop-bands that drastically increases from $\sim 15$ to $\sim 30 \mathrm{~dB} \cdot \mathrm{cm}^{-1}$, while the second refers to the full width half maximum (FWHM) broadening of the discrete spectral stopbands up to a factor of $2 \times$ for specific bands. Indicatively, the $\sim 837 \mathrm{~nm}$ centered band broadens from 16.9 to $32 \mathrm{~nm}$ and the $\sim 950 \mathrm{~nm}$ centered one from 29.7 to $59 \mathrm{~nm}$. The specific spectral features (extinction ratio increase and broadening of bands) are related with the extensive AgI agglomeration promoted by the slow-cooling process (also verified by SEM investigations presented in Figure 1c,d).

The spectral data of Figure 4a,b are of particular interest. Generally, phase and amplitude errors introduced in optical lattices used for distributed light scattering or light confinement (extrinsic etc.) 
result in predominant reduction of the scattering efficiency and broadening of the bandwidth of their spectral signature $[26,27]$. The interesting point observed in the current data refers to the simultaneous spectral broadening of the transmission bandgaps (see Figure 4a,b) and the increase of their extinction ratio (see Figure 4a) for the slow cooled samples; this finding does not agree with the typical cases aforementioned. A plausible explanation can be that the broadening of the bandgaps in the slow cooled FIC infiltrated PCFs comes from their higher refractive index emerging from their thermal history; higher refractive index of the glass strands can justify the spectral shifts of the transmission bands. We speculate that the broadening of the FWHM of the stop-bands for the slow cooling samples is associated with the high refractive index $\mathrm{AgI}$ agglomerated microdomains, that affect the light confinement in these FIC glass infiltrated PCFs. Theoretical and experimental studies performed in photonic bandgap slab waveguides and hollow core photonic crystal fibers which exhibit random disorders of their basic unit cell, resulted in broadening of the bandgap transmission spectrum [28] and violation of the Beer-Lambert law that can describe simple propagation loss mechanisms [29].

Figure 4. Transmission spectra of $1 \mathrm{~cm}$ long $x \mathrm{AgI}+(1-x) \mathrm{AgPO}_{3} / \mathrm{LMA}-10$ fibers fabricated via fast-cooling and slow-cooling methods, for $x=0.3$ (a), and $x=0.55$ (b), i.e., normalized to the corresponding spectrum of the pristine PCF fiber; (c) Absorption and refractive index as obtained from ellipsometry for glass with $x=0.55$; (d) Focus on the short wavelength guiding regime between 450 and $1050 \mathrm{~nm}$ for glass with $x=0.55$, along with ARROW model prediction of the transmission minima [2]. The modal order of the $935 \mathrm{~nm}$ notch is $m=9$.
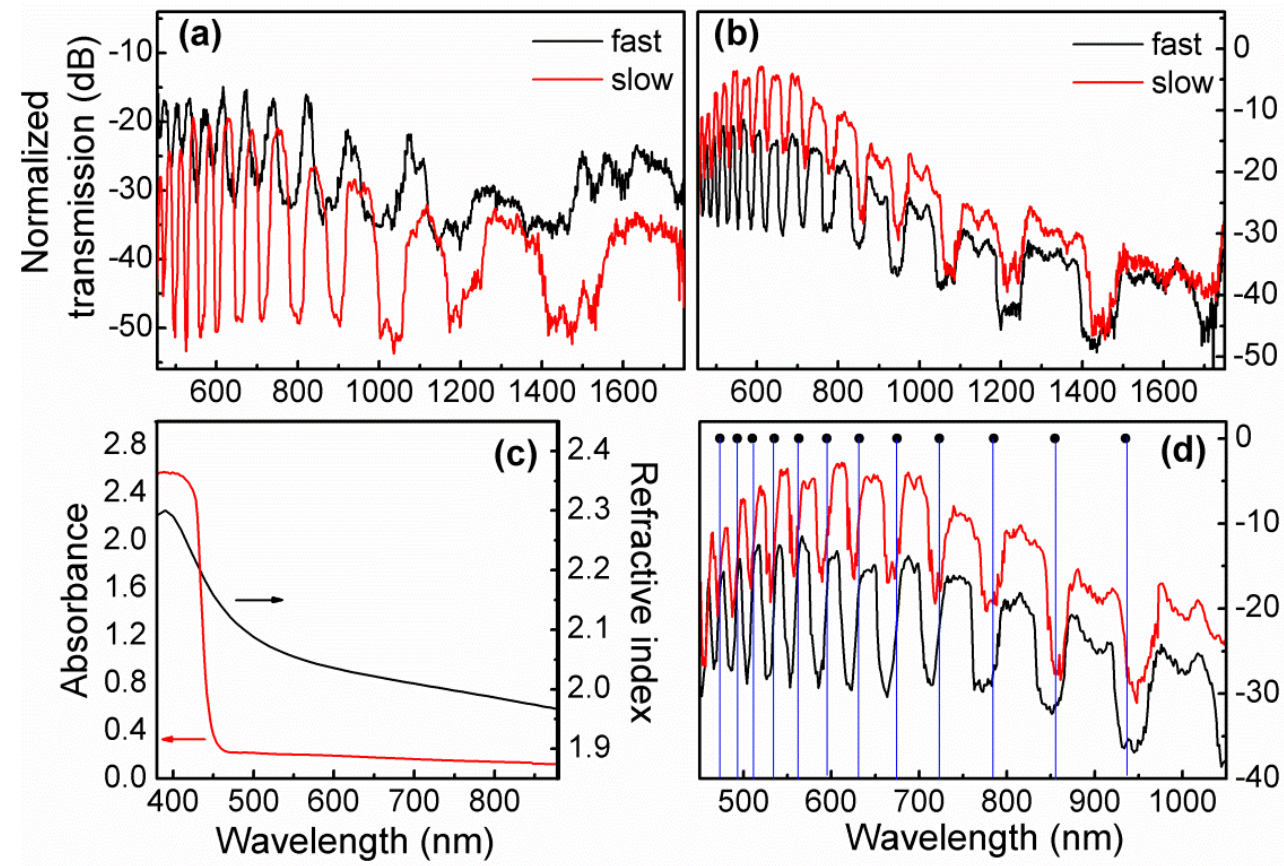

One should also consider that the large size AgI microdomains exhibit wavelength and angular dependent scattering behavior. Simple Mie scattering calculations for a single $300 \mathrm{~nm}$ diameter AgI microdomains assuming a modest $5 \%$ increase of refractive index due to agglomeration, revealed broadband peak scattering spanning from 350 to $1000 \mathrm{~nm}$, approximately [30]; also, strong angular scattering occurred for most of those wavelengths for angles up to $60^{\circ}$. These effects can also affect 
the guiding characteristics of the composite PCF fabricated here. Meanwhile, we are also working further for illustrating the exact physical origin of the spectral broadening of the transmission bands of this PBG guiding PCF.

The effect of the cooling method on the guiding characteristics of the $0.55 \mathrm{AgI}+0.45 \mathrm{AgPO}_{3} / \mathrm{LMA}-10$ fiber is of rather different effect and less prominent compared to the glass with lower AgI concentration. Remarkably, Figure $4 \mathrm{~b}$ reveals that the slow-cooling protocol almost diminishes transmission losses of the composite PCF in the short wavelength guiding regimes, whereas it reduces loss throughout the entire spectral region. We recall now on the corresponding SEM studies of this composite fiber (Figure 2), where even following the slow-cooling approach, the so-formed AgI microdomains did not exceed the size of $\sim 150 \mathrm{~nm}$, despite the fact of higher AgI content compared to glass with $x=0.3$. Normally, in such glass systems, the devitrification and tendency for crystallization is expected close to the highest contents of AgI [31]. However, it is believed that glass formation limits within the constraint environment of the fiber capillaries push the crystallization bias to lower contents of AgI, where glasses exhibit higher glass transition temperatures [14,22], and thus, their ability to adapt to external stresses induced from the much harder silica glass upon cooling is reduced. Additionally, among the fibers of this study, the $0.55 \mathrm{AgI}+0.45 \mathrm{AgPO}_{3} / \mathrm{LMA}-10$ sample exhibits the highest refractive index contrast between the core and the infiltrated medium, which results to significant reduction of mode confinement losses. Thus, as long as it remains free of exceptionally large AgI-clusters, as found to be the case herein independently of the cooling protocol (Figure 2), it is expected to exhibit the most optimum transmission behavior, as it is revealed by experimental measurements of the transmission profile (Figure 4b). Among the two cooling protocols, slow-cooling exhibits even better transmission behavior due to the fact that during the elongated cooling period the residual stresses between silica and the extremely soft FIC glass $\left(T_{\mathrm{g}}=80{ }^{\circ} \mathrm{C}\right.$ for $\left.x=0.55\right)$ vanish, and thus, reducing the loss sources even further.

The lower dependence of the refractive index of the glass melt upon thermal history conditions for the AgI concentration of $x=0.55$, allowed us to use the simple ARROW model to verify the transmission minima of the PBG composite optical fiber (see Figure 4d). Thus, the interpretation of the spectral data of Figure $4 \mathrm{~b}$ using antiguiding ARROW Equation [2], well predicted high loss spectral points, while based on refractive index measurements obtained ellipsometrically for the spectral range between 400 and $1000 \mathrm{~nm}$ (shown in Figure 4c), for a thin glass sample prepared by splat quenching technique. As it is appeared in Figure 4d the agreement of the loss maxima ARROW model data refer to both fast and slow cooling protocols applied in the infiltrated fibers of this specific composition.

Finally, it is worth noting, that for the case of the binary metaphosphate $\mathrm{AgPO}_{3} / \mathrm{LMA}-10$ samples no difference in the transmission profiles was obtained between the two cooling protocols, i.e., fast-cooling and slow-cooling. Moreover, SEM investigations (not shown here) revealed similar quality of $\mathrm{AgPO}_{3}$ glass strands within all LMA-10 studied herein, independently of the cooling method followed during sample fabrication. Such findings are explained in terms of the lower tendency for crystallization and phase separation within the stable silver metaphosphate glass $\mathrm{AgPO}_{3}$ compared to that within AgI-doped FIC glasses. Therefore, in the case of $\mathrm{AgPO}_{3}$ samples, even following the slow cooling protocol does not result to any significant formation of microdomains that will be capable of altering the optical properties of the phosphate glass, and thus, the guiding properties of the composite $\mathrm{AgPO}_{3} / \mathrm{LMA}-10$ PBG fibers. This observation is in total agreement with what has been found in an 
earlier study [7], where sole annealing treatment of $\mathrm{AgPO}_{3}$ glass-infiltrated PCFs showed no alteration on their guiding properties.

\section{Conclusions}

In conclusion, the transmission characteristics of composite $x \mathrm{AgI}+(1-x) \mathrm{AgPO}_{3} /$ silica photonic bandgap (PBG) fibers were tuned by varying $\mathrm{AgI}$ concentration, as well as, following different cooling methods during sample fabrication. As expected, introduction of AgI within the phosphate glass infiltration medium induces additional transmission stop-bands in the PBG spectra profiles of the fibers, along with spectral allocations attributed to the variation of the refractive index contrast between the silica core and the phosphate glass strands. For the lower AgI content $(x=0.3)$, significant differences were observed among the two cooling protocols employed herein, as slow-cooling resulted to the formation of large AgI microdomains within the phosphate glass, which act as centers of scattering and enhance transmission losses. For high AgI content $(x=0.55)$, crystallization within the fiber capillaries was considerably suppressed, even when adopting the slow-cooling protocol, leading to fibers with minimum losses and optimum PBG transmission characteristics. Ongoing work, involves the exploitation of the enhanced charge carrier mobility within fast-ion-conducting glasses, towards tuning the PBG guidance properties of such type of composite fibers upon application of external voltage on the fiber end faces. Also, the realization of fiber waveguiding geometries based on those silver fast-ion-conducting glasses, while utilizing both periodic and highly disordered structures [32] is currently under investigation.

\section{Acknowledgments}

The authors are grateful to Aleka Manousaki (Institute of Electronic Structure and Laser, Foundation for Research and Technology-Hellas) for her assistance with SEM studies, Ioannis Raptis (ThetaMetrisis S.A. and National Center for Scientific Research "Demokritos") for performing optical absorption measurements in the silver fast-ion-conducting glasses, and Eleftherios Iliopoulos (Physics Department, University of Crete) for ellipsometric studies on the same glasses. Both authors would also like to thank the Reviewers for their constructive comments and Georgios Kakarantzas (National Hellenic Research Foundation, Athens) for illustrating discussions. This work is funded by the State Scholarship Foundation (IKY) within the framework of IKY Fellowships of Excellence for Postgraduate Research in Greece Siemens Program, contract number: SR 22091/13.

\section{Author Contributions}

Ioannis Konidakis performed the experimental work (glass synthesis, PCF infiltration, and optical measurements) and wrote the paper. Stavros Pissadakis conceived the idea of the current project, supervised the experimental procedures while offering advice on improving experimental setups, and helped with editing the paper. Data interpretation at all stages was done by both authors, as well as, discussion on future directions and experiments. 


\section{Conflicts of Interest}

The authors declare no conflict of interest.

\section{References}

1. Knight, J.C.; Broeng, J.; Birks, T.A.; Russell, P.S.J. Photonic band gap guidance in optical fibers. Science 1998, 282, 1476-1478.

2. Abeeluck, A.K.; Litchinitser, N.M.; Headley, C.; Eggleton, B.J. Analysis of spectral characteristics of photonic bandgap waveguides. Opt. Express 2002, 10, 1320-1333.

3. Lousteau, J.; Scarpignato, G.; Athanasiou, G.; Mura, E.; Boetti, N.; Olivero, M.; Benson, T.; Sewell, P.; Abrate, S.; Milanese, D. Photonic bandgap confinement in an all-solid tellurite-glass photonic crystal fiber. Opt. Lett. 2012, 37, 4922-4924.

4. Granzow, N.; Uebel, P.; Schmidt, M.A.; Tverjanovich, A.S.; Wondraczek, L.; Russell, P.S. Bandgap guidance in hybrid chalcogenide-silica photonic crystal fibers. Opt. Lett. 2011, 36, 2432-2434.

5. Markos, C.; Yannopoulos, S.N.; Vlachos, K. Chalcogenide glass layers in silica photonic crystal fibers. Opt. Express 2012, 20, 14814-14824.

6. Konidakis, I.; Zito, G.; Pissadakis, S. Photosensitive, all-glass $\mathrm{AgPO}_{3} /$ silica photonic bandgap fiber. Opt. Lett. 2012, 37, 2499-2501.

7. Konidakis, I.; Zito, G.; Pissadakis, S. Silver plasmon resonance effects in $\mathrm{AgPO}_{3} /$ silica photonic bandgap fiber. Opt. Lett. 2014, 39, 3374-3377.

8. Spittel, R.; Kobelke, J.; Hoh, D.; Just, F.; Schuster, K.; Schwuchow, A.; Jahn, F.; Kirchhof, J.; Jäger, M.; Bartelt, H. Arsenic chalcogenide filled photonic crystal fibers. J. Non-Cryst. Solids 2013, 377, 231-235.

9. Kreibig, U. Electronic properties of small silver particles optical-constants and their temperature dependence. J. Phys. F Metal Phys. 1974, 4, 999-1014.

10. Sonnichsen, C.; Franzl, T.; Wilk, T.; von Plessen, G.; Feldmann, J. Plasmon resonances in large noble-metal clusters. New J. Phys. 2002, 4, doi:10.1088/1367-2630/4/1/393.

11. Noginova, N.; Yakim, A.V.; Soimo, J.; Gu, L.; Noginov, M.A. Light-to-current and current-to-light coupling in plasmonic systems. Phys. Rev. B 2011, 84, 35447-35451.

12. Ingram, M.D. Ionic-conductivity in glass. Phys. Chem. Glasses 1987, 28, 215-234.

13. Roling, B.; Ingram, M.D.; Lange, M.; Funke, K. Role of AgI for ionic conduction in $\mathrm{AgI}-\mathrm{AgPO}_{3}$ glasses. Phys. Rev. B 1997, 56, 13619-13622.

14. Sidebottom, D.L. Influence of cation constriction on the ac conductivity dispersion in metaphosphate glasses. Phys. Rev. B 2000, 61, 14507-14516.

15. Bhattacharya, S.; Dutta, D.; Ghosh, A. Dynamics of $\mathrm{Ag}^{+}$ions in $\mathrm{Ag}_{2} \mathrm{~S}$-doped superionic $\mathrm{AgPO}_{3}$ glasses. Phys. Rev. B 2006, 73, 104201-104206.

16. Carini, G.; Cutroni, M.; Fontana, A.; Mariotto, G.; Rocca, F. Inelastic light-scattering in superionic glasses $(\mathrm{AgI})_{x}\left(\mathrm{Ag}_{2} \mathrm{O} \mathrm{Nb}_{2} \mathrm{O}_{3}\right)_{1-x}$. Phys. Rev. B 1984, 29, 3567-3572. 
17. Rousselot, C.; Malugani, J.P.; Mercier, R.; Tachez, M.; Chieux, P.; Pappin, A.J.; Ingram, M.D. The origins of neutron-scattering prepeaks and conductivity enhancement in AgI-containing glasses. Solid State Ion. 1995, 78, 211-221.

18. Markos, C.; Antonopoulos, G.; Kakarantzas, G. Broadband guidance in a hollow-core photonic crystal fiber with polymer-filled cladding. Photonics Technol. Lett. IEEE 2013, 25, 2003-2006.

19. Turitsyn, S.K.; Babin, S.A.; El-Taher, A.E.; Harper, P.; Churkin, D.V.; Kablukov, S.I.; Ania-Castañón, J.D.; Karalekas, V.; Podivilov, E.V. Random distributed feedback fibre laser. Nat. Photonics 2010, 4, 231-235.

20. Mookherjea, S.; Park, J.S.; Yang, S.H.; Bandaru, P.R. Localization in silicon nanophotonic slow-light waveguides. Nat. Photonics 2008, 2, 90-93.

21. Konidakis, I.; Varsamis, C.P.E.; Kamitsos, E.I. Effect of synthesis method on the structure and properties of $\mathrm{AgPO}_{3}$-based glasses. J. Non-Cryst. Solids 2011, 357, 2684-2689.

22. Konidakis, I.; Palles, D.; Varsamis, C.P.E.; Kamitsos, E.I. Influence of synthesis conditions on melt contamination in $\mathrm{AgI}-\mathrm{AgPO}_{3}$ fast ion conducting glasses and structure-property correlations. Unpublished work, 2014.

23. Image J Software. Available online: http://imagej.nih.gov/ij/ (accessed on 7 January 2014).

24. Benassi, P.; Fontana, A.; Rodrigues, P.A.M. Light-scattering in superionic glasses $(\mathrm{AgI})_{x}\left(\mathrm{AgPO}_{3}\right)_{1-x}$ brillouin and raman-scattering. Phys. Rev. B 1991, 43, 1756-1762.

25. Argyros, A.; Birks, T.A.; Leon-Saval, S.G.; Cordeiro, C.M.B.; Russell, P.S. Guidance properties of low-contrast photonic bandgap fibres. Opt. Express 2005, 13, 2503-2511.

26. Feced, R.; Zervas, M.N. Effects of random phase and amplitude errors in optical fiber Bragg gratings. Lightwave Technol. 2000, 18, 90-101.

27. Hughes, S.; Ramunno, L.; Young, J.F.; Sipe, J.E. Extrinsic optical scattering loss in photonic crystal waveguides: Role of fabrication disorder and photon group velocity. Phys. Rev. Lett. 2005, 94, doi:10.1103/PhysRevLett.94.033903.

28. Fokoua, E.N.; Poletti, F.; Richardson, D.J. Analysis of light scattering from surface roughness in hollow-core photonic bandgap fibers. Opt. Express 2012, 20, 20980-20991.

29. Patterson, M.; Hughes, S.; Schulz, S.; Beggs, D.M.; White, T.P.; O’Faolain, L.; Krauss, T.F. Disorder-induced incoherent scattering losses in photonic crystal waveguides: Bloch mode reshaping, multiple scattering, and breakdown of the Beer-Lambert law. Phys. Rev. B 2009, 80, 195305-195310.

30. Bohren, C.F.; Huffman, D.R. Absorption and Scattering of Light by Small Particles; Wiley-VCH: Berlin, Germany, 1998.

31. Tomasi, C.; Mustarelli, P.; Magistris, A.; Ricci, O. Devitrification and phase equilibria in the $x \mathrm{AgI}(1-x) \mathrm{AgPO}_{3}$ system. J. Phys. Chem. B 2002, 106, 2962-2966.

32. Karbasi, S.; Mirr, C.R.; Yarandi, P.G.; Frazier, R.J.; Koch, K.W.; Mafi, A. Observation of transverse Anderson localization in an optical fiber. Opt. Lett. 2012, 37, 2304-2306.

(C) 2014 by the authors; licensee MDPI, Basel, Switzerland. This article is an open access article distributed under the terms and conditions of the Creative Commons Attribution license (http://creativecommons.org/licenses/by/3.0/). 